\title{
Predictive Control Operating at Fixed Switching Frequency of an Induction Machine Fed by a Voltage Source Inverter
}

\author{
Marco Rivera, José Riveros, Consuelo Rodríguez, Patrick Wheeler
}

\begin{abstract}
Predictive torque control (PTC) has been established as one of the viable solutions for variable-speed motor drive control, particularly where a fast response for torque and flux control is required. The main reason for its adoption is the exible architecture and good performance attained with a predictive control scheme. One of the main issues with this approach is the variable switching frequency. Therefore this paper presents an implementation of PTC to overcome this issue. The method consists on the application of two active plus zero voltage vectors to generate a fixed number of switching commutations in the inverter legs during each sampling period. The dwell time for each of the voltage vectors are calculated in the optimization process of the predictive control. The scheme is validated simulation results, demonstrating the constant switching frequency operation whilst keeping the high dynamic torque and ux response.

Keywords-Induction machine, model predictive control, predictive torque control, voltage source inverter.
\end{abstract}

\section{INTRODUCCIÓN}

El control predictivo ha demostrado ser una de las estrategias con mayor potencial accionamientos de velocidad variable [1]. Una de sus aplicaciones es el control directo de torque y flujo (PTC). La implementación clásica de esta técnica presenta las mismas etapa descritas en la teoría del control predictivo [2]; empleando un finito número de alternativas, un modelo predictivo (en este caso particular de torque electromagnético y de flujo del estator), una función de costo (con los errores normalizados de torque y flujo) y la optimización (para seleccionar el próximo estado de conmutación que será empleado). Por las variables controladas, el sistema bajo análisis es más complejo que el considerado en el control predictivo de corriente. No obstante, la regulación del torque y el flujo se consigue con mayor eficiencia con el PTC [3].

El PTC ha sido caracterizado experimentalmente por su elevada velocidad de respuesta de torque y flujo, que lo hace adecuado para usos industriales o en vehículos eléctricos [4]. Además, se deben destacar sus ventajas respecto a su contraparte, el control directo de torque (DTC) [5], tales como la flexibilidad para incluir criterios adicionales en la

Marco Rivera. Director del Laboratorio de Conversión de Energías y Electrónica de Potencia, Universidad de Talca, Curicó, Chile.

José Riveros. Facultad Politécnica, Universidad Nacional de Asunción, Asunción, Paraguay.

Consuelo Rodríguez. Estudiante de Ingeniería Civil Mecatrónica, Facultad de Ingeniería, Universidad de Talca, Curicó, Chile.

Patrick Wheeler. Power Electronics, Machines and Control Group, The University of Nottingham, Nottingham, UK.

978-1-6654-0127-2/21/\$31.00 (c) 2021 IEEE ley de control introduciendo términos en la función de costo (permitiendo su extrapolación a diferentes tipos de convertidores de potencia), el menor rizado de corriente y un ligero incremento en la velocidad de repuesta al emplear modelos más precisos [6]. En contrapartida, el DTC presenta una mayor robustez a variaciones de los parámetros del modelo y menores requerimientos computacionales.

Varios trabajos de investigación continúan enfocándose en el desarrollo de arquitecturas más eficientes del PTC [7]. Entre los desafíos todavía se encuentra la formulación de un procedimiento de sintonización de los factores de peso de la función de costo para la obtención de la respuesta requerida en la aplicación abordada [8], o esquemas con funciones de costo que no requieren del ajuste de los pesos [9]. Otra línea ampliamente considerada es la de los esquemas simplificados para mitigar la elevada carga computacional necesaria para la implementación del PTC [10], así como la supresión del error en régimen estable al carecer de acción integral. Dentro de este conjunto de planteamientos, se puede también destacar la búsqueda de la mitigación del efecto de conmutación a frecuencia variables del PTC, por el elevado costo asociado para soslayar este efecto [11].

En este artículo se presenta una implementación del PTC en un motor de inducción del tipo jaula de ardilla capaz de operar a frecuencia fija de conmutación (igual a la frecuencia de muestreo). Para cumplir con este propósito se ha desarrollado un esquema basado en los principios de operación propuestos en [12] para la regulación de corriente. No obstante, en la función de costo se consideran los errores de flujo y torque electromagnético, por lo que el procedimiento requiere de un mayor análisis al momento de desarrollar el método. Se emplean dos vectores activos en combinación con los ceros para generar el patrón de siete segmentos de conmutación (tal como en la modulación basada en vectores espaciales SVPWM). Sin embargo, el tiempo de permanencia de los vectores se calcula en el proceso de optimización (minimización) de la función de costo. El método es implementado en un entorno de simulación para demostrar el correcto control del torque y flujo, obteniendo respuestas con buena dinámica en el control de la velocidad mecánica del rotor. Todas estas virtudes son obtenidas en conjunto con corrientes del estator con bajos valores de rizado y deformación. 


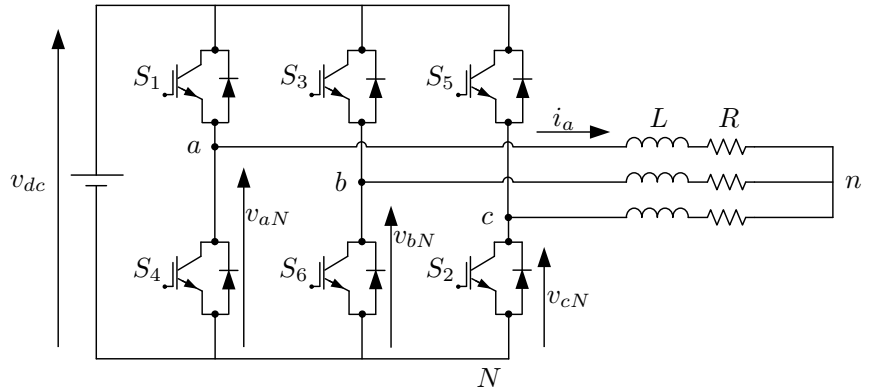

Fig. 1: Circuito inversor fuente de voltaje.

TABLE I: Estados de Conmutación Válidos del VSI

\begin{tabular}{c|cccccc} 
Estado & $S_{1}$ & $S_{2}$ & $S_{3}$ & $S_{4}$ & $S_{5}$ & $S_{6}$ \\
\hline$\# 1$ & 1 & 1 & 0 & 0 & 0 & 1 \\
$\# 2$ & 1 & 1 & 1 & 0 & 0 & 0 \\
$\# 3$ & 0 & 1 & 1 & 1 & 0 & 0 \\
$\# 4$ & 0 & 0 & 1 & 1 & 1 & 0 \\
$\# 5$ & 0 & 0 & 0 & 1 & 1 & 1 \\
$\# 6$ & 1 & 0 & 0 & 0 & 1 & 1 \\
$\# 7$ & 1 & 0 & 1 & 0 & 1 & 0 \\
$\# 8$ & 0 & 1 & 0 & 1 & 0 & 1
\end{tabular}

\section{Modelo Matemático DEL VSI}

La función de un inversor de fuente de voltaje (VSI) es cambiar la señal de voltaje continuo de entrada a una señal de voltaje alterno de salida, con una frecuencia y amplitud variables y determinada por el usuario. La topología del inversor fuente de voltaje básicamente viene representado por la Fig. 1, donde es posible ver que este convertidor de potencia consiste de 6 switches unidireccionales.

Para el correcto funcionamiento de este inversor se debe asegurar que los dos interruptores de cada pierna mostradas en la Fig. 1 deben operar de un modo complementario, con el fin de evitar un cortocircuito de la fuente DC. Como resultado, solo ocho posibles estados de conmutación se permiten para este convertidor, con el cual se generan los voltajes de línea a línea y la corriente circulando en el DC-link. El detalle de estos ocho estados de conmutación válidos, se presenta en la Tabla I.

La corriente del enlace DC $i_{d c}$ se determina como una función de los switches del inversor y las corrientes de salida i como,

$$
i_{d c}=\left[\begin{array}{lll}
S_{1} & S_{3} & S_{5}
\end{array}\right] \mathbf{i}
$$

El voltaje de salida está sintetizado como una función de los switches del inversor y el voltaje del enlace DC $v_{d c}$, como se muestra en la ecuación (2).

$$
\mathbf{v}=\left[\begin{array}{c}
S_{1} \\
S_{3} \\
S_{5}
\end{array}\right] v_{d c}
$$

\section{Modelo Matemático de la Máquina de INDUCCIÓN}

Las ecuaciones de la máquina de inducción considerando un marco de referencia de coordenadas $\alpha \beta$ queda representado por:

$$
\mathrm{v}_{\mathrm{s}}=R_{s} \mathrm{i}_{\mathrm{s}}+d \frac{d \psi_{s}}{d t}+j \omega \psi_{s}
$$

Para la ecuación (4) el voltaje del rotor es igual a 0 , ya que se considera un motor jaula de ardilla.

$$
0=R_{r} \mathrm{i}_{\mathrm{r}}+d \frac{d \psi_{r}}{d t}+j\left(\omega-\omega_{r}\right) \psi_{r}
$$

donde:

- $R_{s}$ y $R_{r}$, corresponden a las resistencias del estator y rotor.

- $i_{\mathrm{s}} \mathrm{y} \mathrm{v}_{\mathrm{s}}$, son los vectores de corriente y voltaje en el estator respectivamente.

- $\mathrm{i}_{\mathrm{r}}$, es el vector de corriente en el rotor.

- $\psi_{s}, \psi_{r}$, son los vectores de flujo del estator y rotor.

- $\omega$ corresponde a la velocidad de rotación en un marco de referencia arbitrario y $\omega_{r}$, corresponde a la velocidad angular del rotor.

El flujo del estator y rotor de la máquina se definen por:

$$
\psi_{s}=L_{s} \mathrm{i}_{\mathrm{s}}+L_{m} \mathrm{i}_{\mathrm{r}}
$$

$$
\psi_{r}=L_{m} \mathrm{i}_{\mathrm{s}}+L_{r} \mathrm{i}_{\mathrm{r}}
$$

Donde $L_{s}, L_{r}$ y $L_{m}$, corresponden a las inductancias del estator, rotor e inductancia de magnetización.

El torque del motor se define como:

$$
T=\frac{3}{2} p R e \psi_{s}^{*} \mathrm{i}_{\mathrm{s}}=-\frac{3}{2} p \operatorname{Re} \psi_{r}^{*} \mathrm{i}_{\mathrm{r}}
$$

donde:

- $T$ y $p$, corresponden al torque electromagnético y al número de pares de polos.

- $\psi^{*}$, es el complejo conjugado de $\psi$.

Las ecuaciones obtenidas se utilizan para estimar el flujo del estator y rotor, además son utilizadas para obtener las corrientes predichas y el flujo de estator y torque eléctrico, utilizando las ecuaciones en tiempo discreto como se mostrará más adelante en el capítulo 6. Las ecuaciones del motor de inducción en función de las variables de estado según [13] quedan definidas como:

$$
\begin{gathered}
\mathrm{i}_{\mathrm{s}}+\tau_{\sigma} \frac{d \mathrm{i}_{\mathrm{s}}}{d t}=-j \omega \tau_{o} \mathrm{i}_{\mathrm{s}}+\frac{K_{r}}{R_{\sigma}}\left(\frac{1}{\tau_{r}}-j \omega_{r}\right) \psi_{r}+\frac{\mathrm{v}_{\mathrm{s}}}{R_{\sigma}} \\
\psi_{r}+\tau_{r} \frac{d \tau_{r}}{d t}=-j(\omega-\omega r) \tau_{r} \psi_{r}+L_{m} \mathrm{i}_{\mathrm{s}}
\end{gathered}
$$

donde:

- $\tau_{r}=\frac{L_{r}}{R_{r}}$

- $\tau_{\sigma}=\sigma \frac{L_{s}}{R_{\sigma}}$

- $\sigma=1-\frac{L_{m}^{2}}{L_{s} L_{r}}$

- $K_{r}=\frac{L_{m}}{L_{r}}$

- $K_{s}=\frac{L_{m}}{L_{s}}$

- $R_{\sigma}=R_{s}+R_{k} K_{R}^{2}$ 


\section{TÉCNICA DE CONTROL PREDictivo PARA LA MÁQUINA DE INDUCCIÓN}

\section{A. Esquema de Control}

El diagrama del control predictivo de torque y flujo operando a frecuencia fija de conmutación está representado en la Fig. 2 [14]. El esquema considera el circuito de potencia del inversor fuente de voltaje y la máquina de inducción. En cada instante de muestreo, un controlador PI es utilizado para manejar la velocidad de la máquina de inducción y es este controlador el que genera la referencia de torque eléctrico. Las mediciones de corriente y velocidad son utilizadas para estimar los flujos de estator y rotor, así como también predecir el comportamiento del flujo y torque para cada uno de los estados válidos del inversor fuente de voltaje. Estas predicciones son luego evaluadas y comparadas con sus respectivas referencias en una función de costo. Se considera además la evaluación de un set de estados de conmutación que generen un torque y flujo lo más cercanos a sus respectivas referencias y la estimación del tiempo óptimo a aplicar cada uno de estos estados de conmutación dentro de un instante de muestreo. El set de estados óptimos y sus respectivos tiempos son aplicados luego al convertidor. A partir de este patrón de conmutación se puede lograr un funcionamiento del convertidor operando a frecuencia fija de conmutación.

\section{B. Ecuación de Predicción}

En cada instante de muestreo $T_{s}$ es necesario obtener las predicciones de torque electromagnético $T_{e}^{p}$ y flujo de estator $\psi_{s}^{p}$ para cada uno de los estados válidos de conmutación del inversor fuente de voltaje para el siguiente instante de tiempo $(k+1)$.

$$
\psi_{s}^{p}(k+1)=\psi_{s}(k)+T_{s} \mathrm{v}_{o}(k+1)-R_{s} T_{s} \mathrm{i}_{o}(k+1)
$$

La expresión para predecir el torque es directamente dependiente del flujo y corriente de estator en base a

$$
T_{e}=\frac{2}{3} p\left(\psi_{s} \times \mathrm{i}_{o}\right)
$$

Teniendo en consideración los valores de flujo y corriente de estator predichos, el torque eléctrico predicho se obtiene como:

$$
T_{e}^{p}(k+1)=\frac{3}{2} p\left[\psi_{s}^{p}(k+1) \times \mathrm{i}_{o}^{p}(k+1)\right]
$$

Es necesario obtener una predicción de la corriente del estator $\mathrm{i}_{o}^{p}(k+1)$, y así calcular una predicción del torque electromagnético. Es por esto que se debe discretizar (8) y reemplazar las derivadas obtenidas por la aproximación basada en Euler, obteniendo una expresión para la predicción de la corriente del estator $\mathrm{i}_{o}^{p}$ en un instante $(k+1)$ :

$$
\begin{aligned}
& \mathrm{i}_{o}^{p}(k+1)=\left(1-\frac{T_{s} r_{\sigma}}{L_{s} \sigma}\right) \mathrm{i}_{o}(k)+ \\
& \frac{T_{s}}{L_{s} \sigma}\left[\left(\frac{k_{r}}{\tau_{r}}-j k_{r} \omega(k)\right) \psi_{\mathrm{r}}(\mathrm{k})+\mathrm{v}_{\mathrm{o}}(\mathrm{k}+1)\right]
\end{aligned}
$$

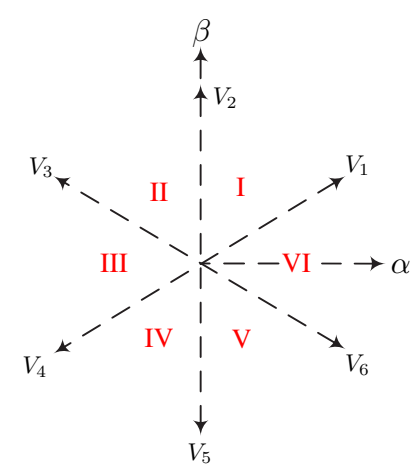

Fig. 3: Representación vectorial para voltajes del convertidor.

Una vez obtenidas las predicciones de flujo de corriente del estator, es posible calcular la predicción del torque electromagnético. Tanto la predicción del torque y flujo del estator se escriben en términos del voltaje del inversor $\mathrm{v}_{s}(k)$. Esto implica que se obtienen siete predicciones diferentes para el torque y el flujo, de acuerdo con el número de vectores de voltaje generados por el VSI.

\section{Función de Costo}

Para el modelo de predicción se define cada vector de voltaje disponible para el VSI en el plano $\alpha \beta$, a través del cual se representan los 7 estados válidos del VSI. Se definen 6 sectores conformados por dos vectores adyacentes que representan los voltajes generados por los estados de conmutación del VSI.

El primer sector se compone por los vectores 1 y 2, que corresponden al voltaje generado por el estado de conmutación 1 y estado de conmutación 2 del inversor. La Figura 3 muestra la representación vectorial descrita anteriormente.

Para la técnica de control predictivo operando a frecuencia fija, se evalúan las predicciones de los dos vectores activos que conforman cada sector del plano $\alpha \beta$ para cada tiempo de muestreo, evaluando la función de costos para cada una de las predicciones, obteniendo así dos funciones de costos $g_{1}$ y $g_{2}$. Además, se obtiene una función de costos $g_{0}$ correspondiente a la predicción obtenida cuando el voltaje es cero.

Las funciones de costos $g_{1}$ y $g_{2}$ son evaluadas por separado en cada uno de los vectores que forman una región. La función $g_{1}$ es evaluada considerando el vector 1 y la función $g_{2}$ es evaluada considerando el vector 2. Posteriormente, estas funciones de costo son evaluadas en una nueva función de costos de la forma que se indica en la ecuación (14). Esta función de costos incluye también los ciclos de trabajo en que se aplicaría cada vector de voltaje activo y el vector cero [15].

$$
g_{(k+1)}=d_{1} g_{1}+d_{2} g_{2}
$$

Estos ciclos son calculados de acuerdo a las siguientes ecuaciones:

$$
\begin{aligned}
& d_{0}=\frac{K}{g_{0}} \\
& d_{1}=\frac{K}{g_{1}}
\end{aligned}
$$




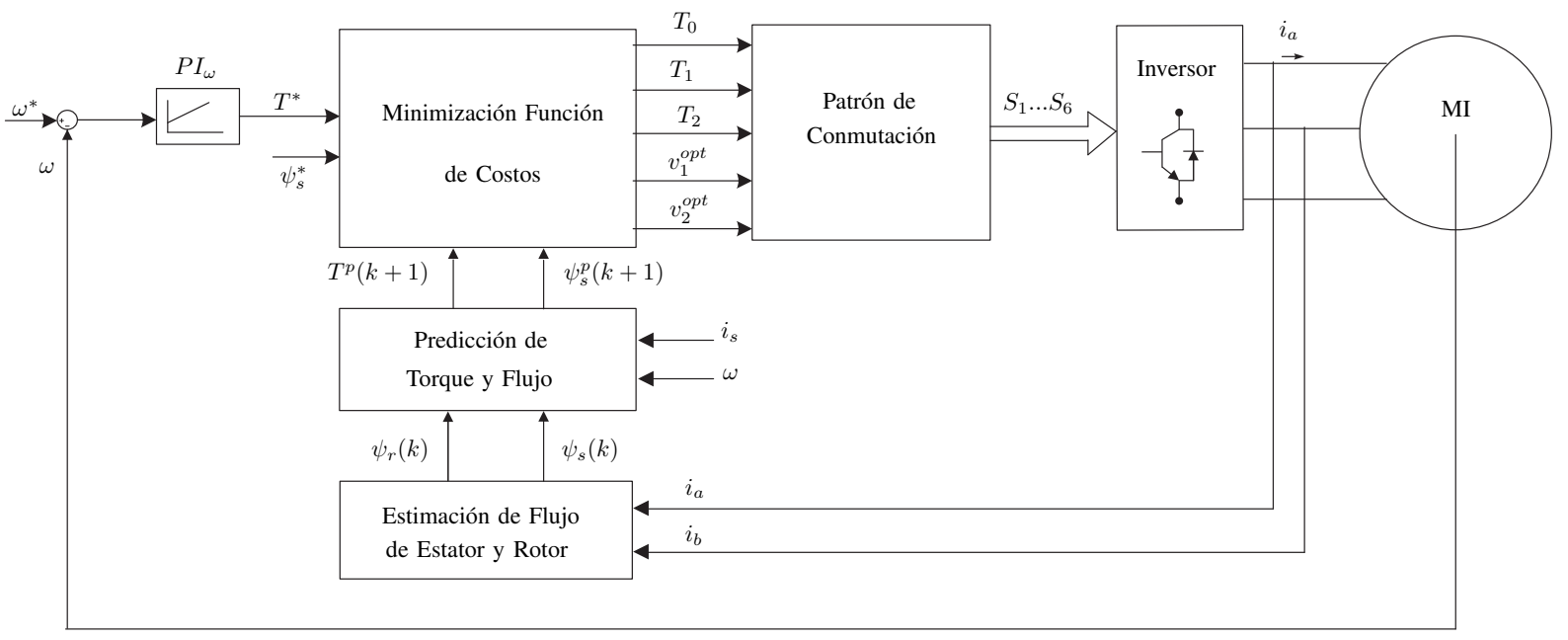

Fig. 2: Esquema de control predictivo a frecuencia fija para un motor de inducción.

$$
\begin{gathered}
d_{2}=\frac{K}{g_{2}} \\
d_{0}+d_{1}+d_{2}=1
\end{gathered}
$$

El ciclo de trabajo $d_{0}$ es evaluado sólo una vez ya que corresponde al vector activo $v_{0}$. Resolviendo las ecuaciones obtenidas anteriormente es posible obtener las expresiones para la constante $K$ y los ciclos de trabajo para cada uno de los vectores:

$$
\begin{gathered}
\frac{K}{g_{0}}+\frac{K}{g_{1}}+\frac{K}{g_{2}}=1 \\
\frac{K g_{1} g_{2}}{g_{0} g_{1} g_{2}}+\frac{K g_{0} g_{2}}{g_{0} g_{1} g_{2}}+\frac{K g_{0} g_{1}}{g_{0} g_{1} g_{2}}=1
\end{gathered}
$$

Donde $K$ corresponde a una constante que relaciona los ciclos de trabajo y las funciones de costo a utilizar en el control. La expresión para la constante $K$ está dada por:

$$
K=\frac{g_{0} g_{1} g_{2}}{g_{1} g_{2}+g_{0} g_{2}+g_{0} g_{1}}
$$

Posteriormente se sustituye la ecuación (21) en (15) , (16) y (17) :

$$
\begin{aligned}
d_{0} & =\frac{g_{1} g_{2}}{g_{1} g_{2}+g_{0} g_{2}+g_{0} g_{1}} \\
d_{1} & =\frac{g_{0} g_{2}}{g_{1} g_{2}+g_{0} g_{2}+g_{0} g_{1}} \\
d_{2} & =\frac{g_{1} g_{0}}{g_{1} g_{2}+g_{0} g_{2}+g_{0} g_{1}}
\end{aligned}
$$

El set de vectores que minimiza la función de costos $g$, serán los que se aplicarán en el siguiente instante de muestreo $(k+1)$.

Los tiempos en que serán aplicados cada uno de los estados óptimos de conmutación (vectores óptimos), $T_{0}, T_{1}$ y $T_{2}$, dentro de un periodo de muestreo $T_{s}$, son determinados por los ciclos de trabajo $d_{0}, d_{1}$ y $d_{2}$ de la siguiente manera:

$$
T_{0}=T_{s} d_{0}
$$

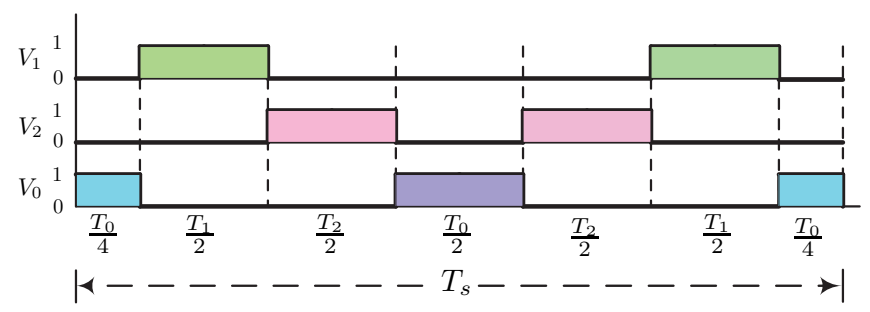

Fig. 4: Patrón de conmutación para control predictivo a frecuencia fija.

$$
\begin{gathered}
T_{1}=T_{s} d_{1} \\
T_{2}=T_{s} d_{2} \\
T_{s}=T_{0}+T_{1}+T_{2}
\end{gathered}
$$

A partir de estos vectores óptimos $v_{1}^{o p t}$ y $v_{2}^{o p t}$ y sus respectivos tiempos en los que serán aplicados $\left(T_{0}, T_{1}\right.$ y $\left.T_{2}\right)$, se establece un patrón de conmutación, que consta de siete pasos tal como se representa en la Fig. 4, generando así una conmutación a frecuencia fija [16]:

1) Se comienza el patrón de conmutación, aplicando un cuarto de su tiempo $T_{0}\left(\frac{T_{0}}{4}\right)$ el vector cero.

2) Posteriormente se procede a aplicar la mitad de su tiempo $T_{1}\left(\frac{T_{1}}{2}\right)$ el primer vector óptimo $v_{1}^{o p t}$.

3) Se aplica la mitad de su tiempo $T_{2}\left(\frac{T_{2}}{2}\right)$ el segundo vector óptimo $v_{2}^{o p t}$.

4) Luego, se aplica el vector cero un periodo equivalente a la mitad de su tiempo $T_{2}\left(\frac{T_{0}}{2}\right)$.

5) Se aplica la mitad de su tiempo $T_{2}\left(\frac{T_{2}}{2}\right)$ el segundo vector óptimo $v_{2}^{o p t}$.

6) Se aplica la mitad de su tiempo $T_{1}\left(\frac{T_{1}}{2}\right)$ el primer vector óptimo $v_{1}^{o p t}$

7) Finalmente se aplica un cuarto de su tiempo $T_{0}\left(\frac{T_{0}}{4}\right)$ el vector cero. 
TABLE II: Parámetros de Simulación del Control Predictivo de Torque de una Máquina de Inducción Alimentada por un VSI.

\begin{tabular}{c|c|c} 
Parámetros & Variables & Valor \\
\hline$T_{s}$ & Tiempo de muestreo & $50[\mu \mathrm{s}]$ \\
$R_{s}$ & Resistencia de estator & $1.35[\Omega]$ \\
$R_{r}$ & Resistencia de rotor & $7.2[\Omega]$ \\
$L_{s}$ & Inductancia de magnetización estator & $0.282[\mathrm{H}]$ \\
$L_{r}$ & Inductancia de magnetización rotor & $0.282[\mathrm{H}]$ \\
$L_{m}$ & Inductancia mutua & $0.286[\mathrm{H}]$ \\
$J$ & Inercia & $0.062\left[\mathrm{Kgm}^{2}\right]$ \\
$p$ & Pares de polos & 2 \\
$p$ & Velocidad nomimal & $1430[\mathrm{rpm}]$ \\
$T_{n o m}$ & Torque nominal & $50[\mathrm{Nm}]$ \\
$T_{l}$ & Torque de carga & $40[\mathrm{Nm}]$ \\
$v_{d c}$ & Voltaje de alimentación & $520[\mathrm{~V}]$ \\
& Tiempo de simulación & $0.9[\mathrm{~s}]$
\end{tabular}

\section{Resultados}

El esquema de control predictivo de torque y flujo operando a frecuencia fija de conmutación de un motor de inducción alimentado por un inversor fuente de voltaje es simulado en Matlab/Simulink, con el fin de validar el comportamiento de la propuesta. La simulación consideró los parámetros indicados en la Tabla II.

Tal como se observa en la Fig. 5, es posible obtener un buen seguimiendo de la velocidad mecánica del motor, demostrando el buen funcionamiento del controlador PI (Fig. 5a). La velocidad de referencia alcanza la velocidad nominal del rotor de $1430[\mathrm{rpm}]$ en el instante de tiempo $\mathrm{t}=0.02$ $[s]$. Cuando el motor comienza a acelerar, logra alcanzar la velocidad nominal a los $0.18[s]$ aproximadamente.

Este controlador PI genera la referencia para el torque eléctrico, cuyo seguimiendo se observa en la Fig. 5b. Aquí se aprecia un torque eléctrico con una rápida respuesta dinámica a su respectiva referencia y con un reducido ripple. Mientras el motor está acelerando hasta el tiempo $\mathrm{t}=0.18[\mathrm{~s}]$, el torque medido se estabiliza siguiendo correctamente el torque de referencia. En el tiempo $t=0.3[s]$ mientras el motor ya está girando a su velocidad nominal, se aplica un torque de carga, presentando una rápida respuesta ante este escalón siguiendo rápidamente el torque de referencia y manteniéndose después de la inversión de marcha ocurrida en el instante $t=0.5[s]$. Igualmente, el flujo de estator sigue a su referencia tal como se indica en la Fig. 5c.

Respecto a las variables del inversor fuente de voltaje, es posible observar en la Fig. 6, para una escala reducida de tiempo [s], el voltaje en el DC-link $v_{d c}$ el cual está establecido en $v_{d c}=520[\mathrm{~V}]$, las corrientes de carga $\mathbf{i}_{\mathbf{o}}$ en coordenadas $\alpha$ $\beta$ [A] con una forma de onda sinusoidal y un muy reducido ripple y el voltaje de carga $v_{a n}$ el cual presenta una forma de onda PWM con un patrón uniforme de conmutación, demostrando la frecuencia fija de conmutación.

\section{CONCLUSIONES}

En este paper se ha presentado la implementación en Matlab/Simulink de una técnica de control predictivo de flujo y torque para un motor de inducción alimentado por un inversor
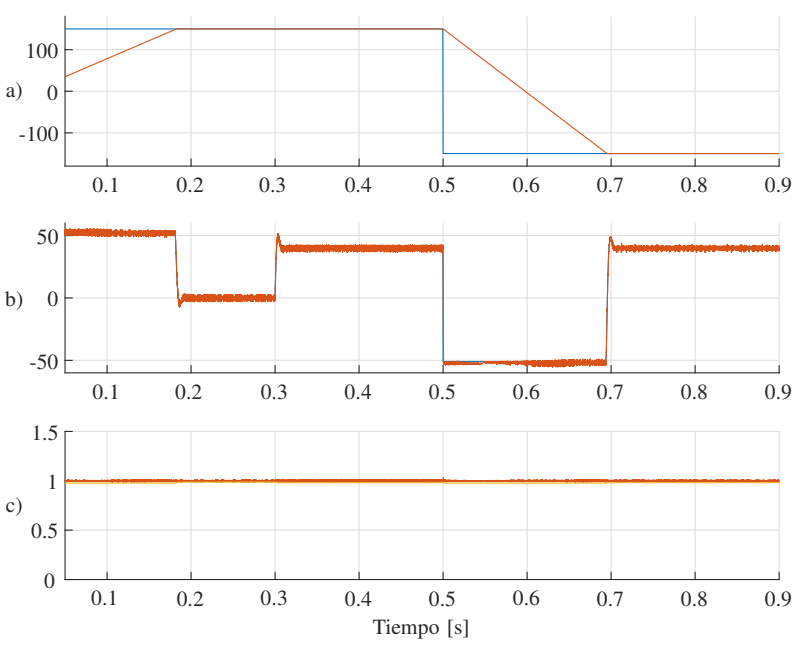

Fig. 5: Resultados de simulación del esquema de control predictivo de torque y flujo a frecuencia fija de conmutación: a) referencia de velocidad y su valor medido [ $\mathrm{rad} / \mathrm{s}]$, b) referencia de torque y su valor medido $[\mathrm{Nm}]$, c) flujo de referencia y estimado del estator [Wb].
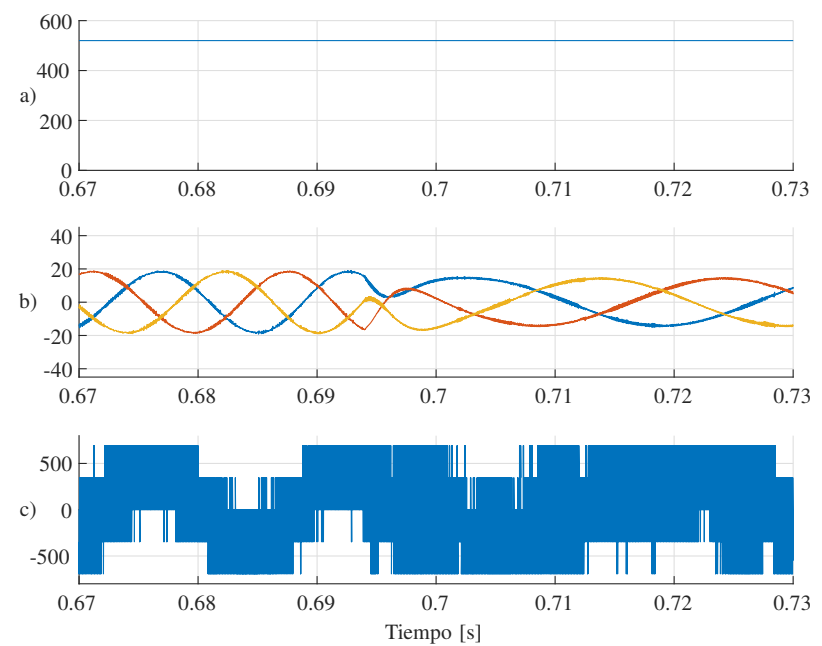

Fig. 6: Resultados de simulación del esquema de control predictivo de torque y flujo a frecuencia fija de conmutación: a) voltaje del enlace DC $v_{d c}[\mathrm{~V}]$, b) corrientes en la salida del VSI $\left.\mathbf{i}_{\mathbf{o}}[\mathrm{A}], \mathrm{c}\right)$ voltaje de salida del convertidor $v_{a n}[\mathrm{~V}]$. 
fuente de voltaje de dos niveles operando con una frecuencia de conmutación fija.

La estrategia considera un modelo de predicción del torque y flujo en base al modelo matemático del convertidor y la máquina de inducción y la selección de un set de estados de conmutación óptimos y sus respectivos tiempos de aplicación, en base a la optimización de una función de costos.

La estrategia es simple de implementar, demostrando buenos resultados en cuando al seguimiento de sus referencias de torque y flujo, con un reducido ripple.

Aspectos tales como un análisis de la estrategia ante cambios en los parámetros y perturbaciones, así como también una comparación cuantitativa con respecto a técnicas tradicionales, y una discusión sobre la sintonización de los diferentes parámetros del control productivo y el controlador PI son considerados como un trabajo futuro.

\section{AGRADECIMIENTOS}

Los autores agradecen al financiamiento del Programa FONDECYT Regular a través del proyecto 1191028, MEC 80190074, MEC 801800974 y FONDAP SERC Chile 15110019.

\section{REFERENCIA}

[1] M. Khosravi, M. Amirbande, D. A. Khaburi, M. Rivera, J. Riveros, J. Rodriguez, A. Vahedi, and P. Wheeler, "Review of model predictive control strategies for matrix converters," IET Power Electronics, vol. 12, no. 12, pp. 3021-3032, 2019.

[2] J. Rodriguez and P. Cortes, Predictive control of power converters and electrical drives. John Wiley \& Sons, 2012, vol. 40.

[3] Y. Zhang, B. Xia, H. Yang, and J. Rodriguez, "Overview of model predictive control for induction motor drives," Chinese Journal of Electrical Engineering, vol. 2, no. 1, pp. 62-76, 2016.

[4] S. Sharma, M. V. Aware, and A. Bhowate, "Symmetrical six-phase induction motor-based integrated driveline of electric vehicle with predictive control," IEEE Transactions on Transportation Electrification, vol. 6, no. 2, pp. 635-646, 2020.

[5] G. S. Buja and M. P. Kazmierkowski, "Direct torque control of pwm inverter-fed ac motors - a survey," IEEE Transactions on Industrial Electronics, vol. 51, no. 4, pp. 744-757, 2004.
[6] F. Wang, Z. Zhang, S. A. Davari, R. Fotouhi, D. Arab Khaburi, J. Rodriguez, and R. Kennel, "An encoderless predictive torque control for an induction machine with a revised prediction model and EFOSMO," IEEE Transactions on Industrial Electronics, vol. 61, no. 12, pp. 66356644, Dec 2014.

[7] P. Karamanakos and T. Geyer, "Model predictive torque and flux control minimizing current distortions," IEEE Trans. Power Electron., vol. 34, no. 3, pp. 2007-2012, March 2019.

[8] P. Guazzelli, W. de Andrade Pereira, C. de Oliveira, A. de Castro, and M. de Aguiar, "Weighting factors optimization of predictive torque control of induction motor by multiobjective genetic algorithm," IEEE Transactions on Power Electronics, vol. 34, no. 7, pp. 6628-6638, July 2019.

[9] F. Wang, H. Xie, Q. Chen, S. A. Davari, J. Rodrguez, and R. Kennel, "Parallel predictive torque control for induction machines without weighting factors," IEEE Transactions on Power Electronics, vol. 35, no. 2, pp. 1779-1788, 2020.

[10] K. M. R. Eswar, K. V. P. Kumar, and T. V. Kumar, "A simplified predictive torque control scheme for open-end winding induction motor drive," IEEE J. of Emerg. Sel. Topics in Power Electron., vol. 7, no. 2, pp. 1162-1172, June 2019.

[11] M. Rivera, D. Faundez, J. Kolar, P. Wheeler, J. A. Riveros, and S. Toledo, "Three-phase rectifiers with current compensation schemes - part i: Passive circuits," in 2018 IEEE International Conference on Electrical Systems for Aircraft, Railway, Ship Propulsion and Road Vehicles International Transportation Electrification Conference (ESARS-ITEC), Nov 2018, pp. 1-6.

[12] F. Donoso, A. Mora, R. Cardenas, A. Angulo, D. Saez, and M. Rivera, "Finite-set model-predictive control strategies for a 3L-NPC inverter operating with fixed switching frequency," IEEE Transactions on Industrial Electronics, vol. 65, no. 5, pp. 3954-3965, May 2018.

[13] Predictive control of power converters and electrical drives. John Wiley \& Sons, 2012, ch. Predictive Control of Induction Machines.

[14] F. Donoso, A. Mora, R. Crdenas, A. Angulo, D. Sez, and M. Rivera, "Finite-set model-predictive control strategies for a 31-npc inverter operating with fixed switching frequency," IEEE Transactions on Industrial Electronics, vol. 65, no. 5, pp. 3954-3965, 2018.

[15] M. Ayala, J. Rodas, R. Gregor, J. Doval-Gandoy, O. Gonzalez, M. Saad, and M. Rivera, "Comparative study of predictive control strategies at fixed switching frequency for an asymmetrical six-phase induction motor drive," in 2017 IEEE International Electric Machines and Drives Conference (IEMDC), 2017, pp. 1-8.

[16] R. E. Prez-Guzmn, M. Rivera, N. Vicencio, and P. W. Wheeler, "Model predictive control in three-phase inverters," in 2019 IEEE CHILEAN Conference on Electrical, Electronics Engineering, Information and Communication Technologies (CHILECON), 2019, pp. 1-6. 